\section{Optical coherence tomography in a patient with tobacco-alcohol amblyopia}

\begin{abstract}
Aim To report optical coherence tomography (OCT) finding in a patient with tobacco-alcohol amblyopia.

Methods A 45-year-old man presented with a gradual decrease in vision over 4 years. He had smoked a half to one pack of cigarettes per day and had consumed $350 \mathrm{cc}$ of gin per day for 30 years. A detailed ophthalmologic examination was performed.

Results His corrected visual acuities were 20/ 800 OD and 20/200 OS. A Goldmann visual field examination showed ceco-central scotomas in both eyes. OCT using a peripapillary Fast RNFL (retinal nerve fiber layer) programme showed a small decrease in the RNFL thickness of the superotemporal quadrant in the normative diagram of the right eye in spite of a markedly increased RNFL thickness in both eyes.

Conclusion During the phase of visual loss in a patient with tobacco-alcohol amblyopia, visual loss may precede optic disc changes as detected by OCT.

Eye (2008) 22, 469-470; doi:10.1038/sj.eye.6702821; published online 13 April 2007
\end{abstract}

Keywords: optical coherence tomography; OCT; tobacco-alcohol amblyopia; toxic-nutritional optic neuropathy; retinal nerve fiber layer; RNFL

\section{Introduction}

Tobacco-alcohol amblyopia or toxic-nutritional optic neuropathy is characterized by decreased visual acuity, central or cecocentral scotoma, and dyschromatopsia in patients who abuse tobacco and alcohol. ${ }^{1,2}$ We could find no reference to an optical coherence tomography (OCT) finding in a patient with tobacco-alcohol amblyopia utilizing MEDLINE.

C Kee ${ }^{1}$ and J-M Hwang ${ }^{2}$

Case report

A 45-year-old man presented with a gradual decrease in vision over 4 years. He had smoked a half to one pack of cigarettes per day for 30 years and had consumed $350 \mathrm{ml}$ of Korean gin ( $21 \%$ alcohol) per day over the same period. $\mathrm{He}$ denied taking any medications over the previous few months. He admitted not eating when he had been drinking 4-5 bottles of Korean gin per day for 10-14 days on each occasion once or twice a year for the past 4 years. The family history was unremarkable.

His corrected visual acuities were 20/800 OD and 20/200 OS. He showed orthotropia at distance and two prism diopters of exophoria at near in the primary position. Ductions and versions were full. His pupils were isocoric and reactive to light, and his fundoscopic examination was normal except for a horizontally increased cup/disc of 0.5 . A Goldmann visual field examination showed cecocentral scotoma in both eyes. OCT, (Carl Zeiss Meditec, Dublin, USA) using a peripapillary fast retinal nerve fibre layer (RNFL) program, showed a small decrease in the RNFL thickness of the superotemporal quadrant in the normative diagram of the right eye (Figure 1, arrowhead) inspite of a markedly increased RNFL thickness in both eyes (Figure 1, arrow).

\section{Comment}

OCT quantitatively assesses retinal and optic nerve thickness and provides accurate and reliable RNFL thickness measurements. ${ }^{3}$ In our patient, with tobacco-alcohol amblyopia, OCT showed a markedly increased RNFL thickness in both eyes as well as a small decrease in RNFL thickness of the superotemporal quadrant in the right eye. Increased RNFL thickness is consistent with OCT findings in a case of methanol poisoning with severe peripapillary nerve fibre
1 Department of Ophthalmology, Samsung Medical Center, School of Medicine Sungkyunkwan University, Seoul, Korea

${ }^{2}$ Department of Ophthalmology, Seoul National University College of Medicine, Seoul National University Bundang Hospital, Sungnam, Korea

Correspondence:

J-M Hwang,

Department of

Ophthalmology, Seoul National University College of Medicine, Seoul National University Bundang Hospital, 300, Gumi-dong, Bundang-gu, Sungnam, Gyeong-gi 463-707, Korea Tel: + 8231787 7372; Fax: + 82317874057

E-mail: hjm@snu.ac.kr

Received: 11 December 2006

Accepted in revised form: 9 March 2007 Published online: 13 April 2007 

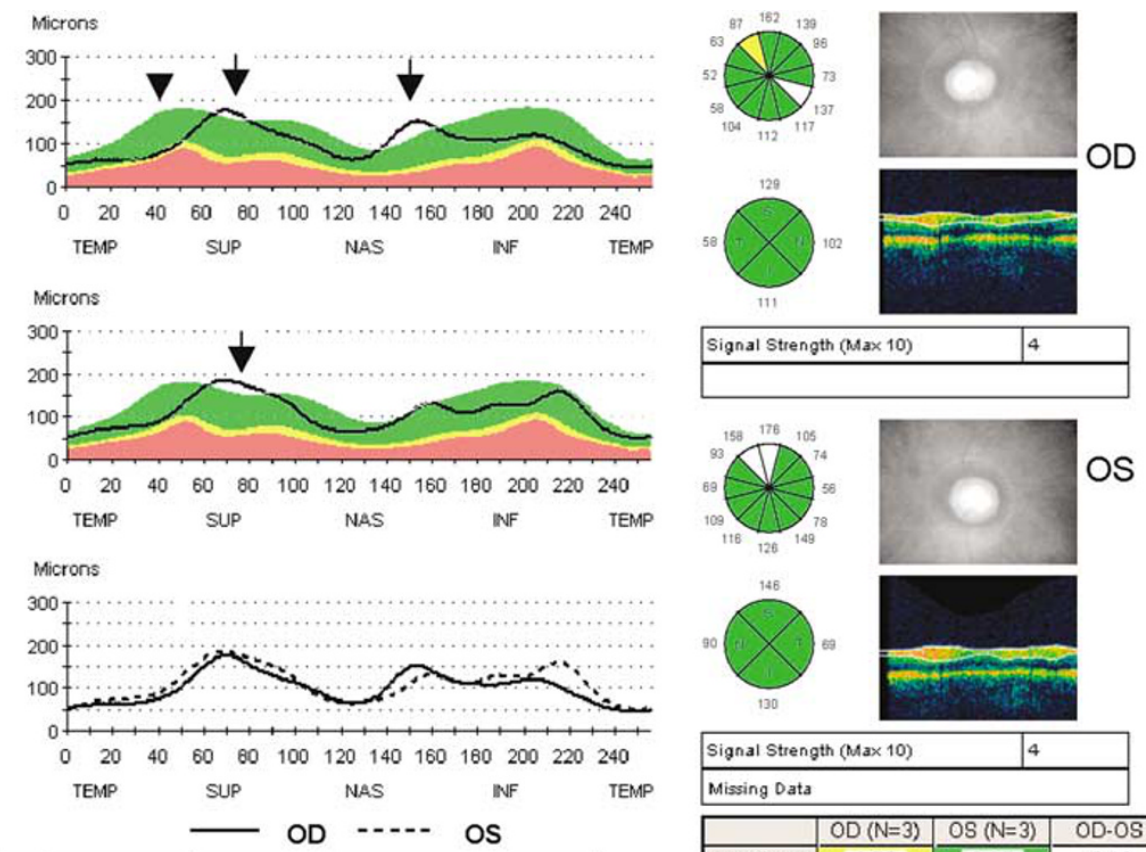

\begin{tabular}{|c|c|c|c|}
\hline \multicolumn{2}{|c|}{ Signal Strength $(\operatorname{Max} 10)$} & \multicolumn{2}{|c|}{4} \\
\hline \multicolumn{4}{|l|}{ Missing Data } \\
\hline & $\mathrm{OD}(\mathrm{N}=3)$ & $\operatorname{OS}(\mathrm{N}=3)$ & OD-OS \\
\hline ImaxiSmax & 0.75 & 0.87 & -0.12 \\
\hline Smaxilmax & 1.33 & 1.14 & 0.19 \\
\hline Smavoravg & 3.06 & 2.65 & 0.40 \\
\hline Imax/Tavg & 2.30 & 2.32 & -0.02 \\
\hline SmavoNavg & 1.73 & 2.04 & -0.31 \\
\hline Max-Min & 132.00 & 134.00 & -2.00 \\
\hline Smax & 176.00 & 184.00 & -8.00 \\
\hline Imax & 132.00 & 161.00 & -29.00 \\
\hline Savg & 129.00 & 146.00 & -17.00 \\
\hline lavg & 111.00 & 130.00 & -19.00 \\
\hline Avg. Thick & 99.94 & 109.01 & -9.07 \\
\hline
\end{tabular}

Figure 1 Optical coherence tomography of nerve fibre layer thickness. Small decrease in the retinal nerve fibre thickness of the superotemporal quadrant in the normative diagram of the right eye (arrowhead). Markedly increased retinal nerve fibre layer thickness in both eyes (arrows).

swelling and intraretinal fluid accumulation, ${ }^{4}$ and may be explained with a pathologic findings of mitochondrial swelling and vacuolation in the retinal pigment epithelium, photoreceptor inner segments, and optic nerve verified in a rodent model. ${ }^{5} \mathrm{~A}$ small decrease in RNFL thickness of the superotemporal quadrant is also consistent with a decreased RNFL thickness in a case of methanol poisoning on repeated OCT 2 years later. In our patient, a true extent of RNFL reduction was probably masked by RNFL oedema. OCT follow-up in our patient may reveal similar changes with a case with methanol poisoning.

In conclusion, during the acute phase of visual loss in a patient with tobacco-alcohol amblyopia, visual loss may precede optic disc changes as detected by fundoscopic examinations or by OCT.

\section{Acknowledgements}

This work was supported by Grant No. R01-2005-00010875-0 from the Basic Research Program of the Korea
Science and Engineering Foundation. None of the authors has a financial interest in any material or method in the article.

\section{References}

1 Woon C, Tang RA, Pardo G. Nutrition and optic nerve disease. Semin Ophthalmol 1995; 10: 195-202.

2 Potts AM. Tobacco amblyopia. Surv Ophthalmol 1973; 117: 313-339.

3 Schuman JS, Hee MR, Puliafito CA, Wong C, PedutKloizman T, Lin CP et al. Quantification of nerve fiber layer thickness in normal and glaucomatous eyes using optical coherence tomography. Arch Ophthalmol 1995; 113: 586-596.

4 Fujihara M, Kikuchi M, Kurimoto Y. Methanol-induced retinal toxicity patient examined by optical coherence tomography. Jpn J Ophthalmol 2006; 50: 239-241.

5 Eells JT, Henry MM, Lewandowski MF, Seme MT, Murray TG. Development and characterization of a rodent model of methanol induced retinal and optic nerve toxicity. Neurotoxicology 2000; 21: 321-330. 